\title{
TRANSICIÓN JUSTA Y LUCHA CONTRA EL CAMBIO CLIMÁTICO EN EL PACTO VERDE EUROPEO EN EL PROYECTO DE LEY DE CAMBIO CLIMÁTICO EN ESPAÑA
}

\author{
Henar Álvarez Cuesta \\ Profesora TU Derecho del Trabajo y de la Seguridad Social \\ Universidad de León
}

\begin{abstract}
La lucha contra el cambio climático sigue constituyendo uno de los mayores desafíos de la sociedad y ha de ser abordado también desde una perspectiva iuslaboralista. El cambio de modelo productivo diseñado desde la Unión Europea mediante el Pacto Verde europeo y asumido por el Proyecto de Ley de Cambio Climático y Transición Energética ha de llevarse a cabo respetando los derechos sociales para conseguir una transición justa hacia un desarrollo sostenible y un trabajo decente. Para ello, la investigación desarrollada examina los sectores y actividades afectados y las herramientas propuestas para detectar con antelación los elementos clave a los cuales habrá de prestarse atención desde el diálogo social y la negociación colectiva para anticipar los cambios y evitar errores.
\end{abstract}

The fight against climate change continues to be one of the greatest challenges in society and must also be approached from a labor-related perspective. The change in the production model designed by the European Union through the European Green Pact and assumed by the Draft Law on Climate Change and Energy Transition must be carried out respecting social rights to achieve a just transition towards sustainable development and decent work. . To do this, the research carried out examines the sectors and activities affected and the tools proposed to detect in advance the key elements to which attention will have to be paid from social dialogue and collective bargaining to anticipate changes and avoid mistakes.

Palabras clave: cambio climático, transición justa, formación, Pacto Verde, empleo Key words: climate change, fair transition, training, Green Deal, employment

IUSLabor 2/2020, ISSN 1699-2938, p. 74-100.

DOI. 10.31009/IUSLabor.2020.i02.04

Fecha envío: 28.5.2020 | Fecha aceptación: 10.6.2020 


\section{Sumario}

1. El desafío de la lucha contra el cambio climático

2. El Pacto Verde europeo

2.1 Sectores e industrias implicadas en el Pacto Verde

2.2 Dimensión social del Pacto Verde: la transición justa

3. La lucha contra el cambio climático en España

4. Conclusiones

5. Bibliografía 


\section{El desafío de la lucha contra el cambio climático}

Los retos a los que se enfrenta el mundo en la actualidad no tienen precedentes (y la reciente pandemia causada por la COVID19 dan buena prueba de ello). Una emergencia climática mundial, una pérdida catastrófica de biodiversidad y la contaminación del aire, del agua y del suelo socavan las bases de la prosperidad futura en Europa y en todo el mundo y las repercusiones de tales desafíos representan una amenaza apremiante para la salud humana y la viabilidad de nuestras cadenas alimentarias y sistemas agrícolas. En un planeta degradado, no habrá vida, ni empleo, ni empresas ${ }^{1}$.

Frente al panorama descrito, las estrategias diseñadas, expuestas infra, consisten en la mitigación en las emisiones de gases de efecto invernadero; en la previsión de tácticas de adaptación al cambio en las zonas más vulnerables a los impactos del cambio climático; y en la prevención/adaptación. Esta última incluye aquellas acciones que reducen el cambio climático a través de medidas de control de emisiones de gases de efecto invernadero. Dentro de la adaptación ${ }^{2}$, cabe distinguir dos tipos: reactiva, una vez manifestados los cambios, o anticipatoria, bien planificada y emprendida antes de que sean evidentes ${ }^{3}$. No es preciso explicar prolijamente cómo resulta más eficaz la llevada a cabo de forma anticipada y a largo plazo, aun cuando quizá no nos encontremos ante tal opción ya ${ }^{4}$.

Las vías descritas conducen a un cambio hacia una economía con menor emisión de carbono, que provocará nuevos desafíos a los modelos legales existentes, se ejercerán nuevas presiones sobre la ocupación, el ajuste y las estrategias de capacitación, y los lugares de trabajo industriales de alta producción de carbono pueden verse afectados de manera impredecible. Entre los actores clave de las relaciones laborales (gobiernos, empleadores, trabajadores y sindicatos $)^{5}$ habrá ganadores y perdedores, así como en los mercados laborales nacionales e internacionales ${ }^{6}$.

\footnotetext{
${ }^{1}$ Dictamen del Comité Económico y Social Europeo sobre «La economía sostenible que necesitamos» (2020/C 106/01).

${ }^{2}$ Autores Varios, Climate change and labour: impacts of heat in the workplace, UNDP, 2016, p. 26.

${ }^{3}$ Instituto Sindical de Trabajo, Ambiente y Salud (ISTAS), ¿Cómo afecta el cambio climático a la salud humana?, CC.OO./ISTAS, 2016, p. 27.

${ }^{4}$ Álvarez Cuesta, Henar, "El contenido social en las leyes contra el cambio climático", en La Gouvernance et l'enjeu de concilier l'èthique et la performance, Pamplona, Eunsa, 2018, p. 279 y ss.

${ }^{5}$ DUPRESSOIR, Sophie et alii, Climate Change and employment: Impact on employment in the European Union-25 of climate change and CO2 emission reduction measures by 2030, Brussels, Wuppertal Institute, 2007.

${ }^{6}$ Doorey, David, "Just Transitions Law: Putting Labour Law to Work on Climate Change", Journal of Environmental Law and Practice, núm. 30, 2017, p. 3.
} 
Por todo ello, el Derecho del Trabajo no puede permanecer ajeno ni ausente ${ }^{7}$. El cambio climático $^{8}$ merece atención desde esta perspectiva asumiendo dos visiones complementarias: de un lado, los efectos que acarrea para el empleo en numerosos sectores $^{9}$; de otro, las consecuencias que las estrategias de lucha o adaptativas conllevan para determinadas actividades $\mathrm{y}$, en consecuencia, para quienes prestan servicios en cualquiera de ellos. De hecho, la crisis ecológica y la social están íntimamente vinculadas ${ }^{10}$, y la repercusión es mayor en los países o colectivos más pobres, pues "la vulnerabilidad al cambio climático es una consecuencia directa de la pobreza"11 y los empleos más afectados resultarán aquellos con salarios más bajos y condiciones laborales más precarias.

A nivel mundial, "dinamita" los objetivos de desarrollo sostenible ${ }^{12}$, en particular dificulta el logro de los siguientes:

1. Erradicar la pobreza en todas sus formas en todo el mundo.

2. Poner fin al hambre, conseguir la seguridad alimentaria y una mejor nutrición, y promover la agricultura sostenible.

3. Garantizar una vida saludable y promover el bienestar para todos y todas en todas las edades.

4. Alcanzar la igualdad entre los géneros y empoderar a todas las mujeres y niñas.

5. Garantizar la disponibilidad y la gestión sostenible del agua y el saneamiento para todos.

6. Asegurar el acceso a energías asequibles, fiables, sostenibles y modernas para todos.

7. Fomentar el crecimiento económico sostenido, inclusivo y sostenible, el empleo pleno y productivo, y el trabajo decente para todos ${ }^{13}$.

\footnotetext{
${ }^{7}$ No lo ha hecho, baste destacar el magnífico estudio en ChaCARTEGUI JÁVEGA, Consuelo, Negociación colectiva y sostenibilidad medioambiental: un compromiso social y ecológico, Albacete, Bomarzo, 2018.

${ }^{8}$ La Convención Marco de las Naciones Unidas sobre el Cambio Climático entiende por cambio climático un cambio de clima atribuido directa o indirectamente a la actividad humana que altera la composición de la atmósfera mundial y que se suma a la variabilidad natural del clima observada durante períodos de tiempo comparables; y por sus efectos adversos, los cambios en el medio ambiente físico o en la biota resultantes del cambio climático que tienen efectos nocivos significativos en la composición, la capacidad de recuperación o la productividad de los ecosistemas naturales o sujetos a ordenación, o en el funcionamiento de los sistemas socioeconómicos, o en la salud y el bienestar humanos (artículo 1).

9 Al respecto, Álvarez CUESTA, Henar, Empleos verdes. Una aproximación desde el Derecho del Trabajo, Albacete, Bomarzo, 2016, p. 17 y ss.

10 Monereo Pérez, Jose Luis y Rivas Vallejo, Pilar, Prevención de riesgos laborales y medio ambiente, Granada, Comares, 2008, p. 34.

${ }^{11}$ RosemberG, Anabella, "Llevar a cabo una transición justa. Las conexiones entre el cambio climático y el empleo, y propuestas para futuras investigaciones”, Boletín Internacional de Investigación Sindical, núm. 2, vol. 2, 2010, p. 147.

${ }^{12}$ Autores Varios, Climate change and labour: impacts of heat in the workplace, cit., p. 24.
} 
8. Reducir las desigualdades entre países y dentro de ellos.

De hecho, combatir la crisis climática constituye no solo uno de dichos objetivos (13.Tomar medidas urgentes para combatir el cambio climático y sus efectos), sino parte fundamental de otros dos:

14. Conservar y utilizar de forma sostenible los océanos, mares y recursos marinos para lograr el desarrollo sostenible.

15. Proteger, restaurar y promover la utilización sostenible de los ecosistemas terrestres, gestionar de manera sostenible los bosques, combatir la desertificación y detener y revertir la degradación de la tierra, y frenar la pérdida de diversidad biológica.

En cuanto hace a las repercusiones de la crisis climática en España, numerosos estudios "coinciden en señalar a la región mediterránea como una de las áreas del planeta más vulnerables frente al cambio climático". Por su situación geográfica y sus características socioeconómicas, "se enfrenta a importantes riesgos derivados del cambio climático que inciden directa o indirectamente sobre un amplísimo conjunto de sectores económicos y sobre todos los sistemas ecológicos españoles, acelerando el deterioro de recursos esenciales para nuestro bienestar como el agua, el suelo fértil o la biodiversidad y amenazando la calidad de vida y la salud de las personas" (Preámbulo Proyecto de Ley de Cambio Climático) ${ }^{14}$.

Ante la certeza de la amenaza (o las consecuencias ya presentes), en términos de pérdida de empleo y cohesión social, y las migraciones (ya se ha comenzado a hablar de refugiados medioambientales) que está provocando el cambio climático, hacen necesario diseñar un proceso de transformación a otro modelo productivo en el que se aúnen ecología y empleo ${ }^{15}$. Este cambio ha de llevarse a cabo modificando normas, pero respetando los derechos sociales en aras a conseguir una transición justa hacia un desarrollo sostenible y un trabajo decente. El concepto acuñado para ello ("transición

\footnotetext{
${ }^{13}$ QuinTERo LimA, María Gemma, "ODS 8: trabajo decente y el futuro del trabajo, Tiempo de paz, núm. 132, 2019, pp. 68 y ss. y OIT, Time to act for SDG 8. Integrating Decent Work, Sustained Growth and Environmental Integrity, Ginebra, OIT, 2019.

${ }^{14} \mathrm{Al}$ respecto, basta revisar los datos que figuran en el Plan Nacional de Adaptación al Cambio Climático 2021-2030 Borrador 30 abril 2020, pp. 13 y ss. Desde otra perspectiva McKinsey Global Institute: Climate risk and response. Physical hazards and socioeconomic impacts, enero 2020.

${ }^{15}$ OIT, The social and decent work dimensions of a new Agreement on Climate Change, Ginebra, OIT, 2009, p. 9-10.
} 
justa") pretende contribuir a los objetivos de trabajo decente para todos, inclusión social y erradicación de la pobreza ${ }^{16}$.

Dicha transición justa cabe definirla como un cambio hacia economías inclusivas y con bajas emisiones de carbono, que, al tiempo, maximice las oportunidades de prosperidad económica, justicia social, derechos y protección social para todos, sin dejar a nadie atrás. La hipótesis de trabajo radica en que "las transformaciones en las decisiones y medidas de orden económico, social, tecnológico y político pueden posibilitar las trayectorias resilientes al clima"17 y conseguir empleos verdes y decentes ${ }^{18}$. En consecuencia, es preciso aunar los conceptos mencionados ${ }^{19}$ : "el trabajo prestado por los asalariados sea indigno por participar en la degradación medioambiental, como imposibilitar que -precisamente en origen- se pongan en práctica actuaciones empresariales, no sólo atentatorias de la salud de los propios trabajadores, sino también las que pudieran conllevar menoscabo ambiental"20.

El propio Acuerdo de París de 2015, el desarrollo de sus reglas en Katowice y la Agenda 2030 para el Desarrollo Sostenible marcan el inicio de una agenda global hacia el desarrollo sostenible, que conlleva la transformación del modelo económico y de un nuevo contrato social de prosperidad inclusiva dentro de los límites del planeta. Por ello, el propio Acuerdo de París establece el imperativo de una transición justa como uno de los elementos esenciales de la acción climática ${ }^{21}$.

El Parlamento europeo utiliza al respecto el concepto de "economía del bienestar", la cual debe proteger los ecosistemas, conservar la biodiversidad, lograr una transición justa hacia una forma de vida climáticamente neutra en toda la UE y fomentar el emprendimiento sostenible ${ }^{22}$.

${ }^{16}$ OIT, Guidelines for a just transition towards environmentally sustainable economies and societies for all, Ginebra, OIT, 2015, p. 6.

${ }^{17}$ Autores Varios, Cambio climático 2014 Impactos, adaptación y vulnerabilidad. Contribución del Grupo de trabajo II al Quinto Informe de Evaluación del Grupo Intergubernamental de Expertos sobre el Cambio Climático, 2014, p. 28.

${ }^{18}$ LÓPEZ RODRíGUEZ, Josune, "The promotion of both decent and Green jobs through cooperatives", Boletín de la Asociación Internacional de Derecho Cooperativo, núm. 54, 2019, p. 115 y ss.

${ }^{19}$ Así lo hace la OIT, Directrices de política para una transición justa hacia economías y sociedades ambientalmente sostenibles para todos, Ginebra, OIT, 2015.

${ }^{20}$ PÉREZ AMORÓs, Francisco, "Derecho del trabajo y medio ambiente: unas notas introductorias", Gaceta Laboral, vol. 14, 2010, p. 190.

${ }^{21}$ DoOREY, David, “Just Transitions Law: Putting Labour Law to Work on Climate Change”, cit., p. 4.

${ }^{22}$ Dictamen del Comité Económico y Social Europeo sobre «La economía sostenible que necesitamos» (2020/C 106/01). 
Ya resulta un lugar común hablar del impacto de la tecnología (automatización, robotización) en el empleo, y dividir las opiniones en tecnooptimistas o tecnopesimistas. Las medidas de lucha y adaptación al cambio climático tendrán (tienen) impacto también en el volumen de empleo y las características del mismo, pero presenta una diferencia trascendental. Mientras todavía se desconocen los nuevos cambios que traerá aparejada la tecnología en el futuro, cabe realizar una estimación más precisa de las consecuencias que las normas de lucha contra el cambio climático acarrean, así como de las implicaciones de la crisis climática ya desencadenadas y de aquellas que deberemos asumir si no combatimos las causas de la emergencia climática.

Siguiendo la reflexión, en las páginas siguientes se van a examinar las líneas maestras acuñadas desde la Unión Europea y desde el ámbito interno en orden a luchar contra el cambio climático y calibrar el impacto en los diferentes sectores afectados, así como analizar las medidas destinadas a propiciar la mentada transición justa.

\section{EI Pacto Verde europeo}

Ante el reto inaplazable, la Unión Europea, a través del Pacto Verde europeo (apostando por la vía del Green Deal y no por el decrecimiento), trata de diseñar una nueva estrategia de crecimiento con el objetivo de transformar la Unión Europea en una "sociedad equitativa y próspera, con una economía moderna, eficiente en el uso de los recursos y competitiva, en la que no habrá emisiones netas de gases de efecto invernadero en 2050 y el crecimiento económico estará disociado del uso de los recursos"23. También el Consejo Económico y Social Europeo ha abogado por un nuevo pacto ecológico y social europeo que logre la inversión a gran escala necesaria para asegurar una transición justa hacia una economía climáticamente neutra y cree puestos de trabajo de calidad en todos los territorios ${ }^{24}$.

Este Pacto Verde utiliza como pilar la idea de sostenibilidad y economía circular unidas a la transición justa e integradora (la necesidad de una transición justa, cuyos costes y beneficios se distribuyan equitativamente entre los distintos grupos sociales, las industrias y las regiones y entre las generaciones presentes y futuras ${ }^{25}$ ), y aplica también la Agenda 2030 y los Objetivos de Desarrollo Sostenible de las Naciones Unidas. A este

\footnotetext{
${ }^{23}$ Comunicación de la Comisión al Parlamento Europeo, al Consejo Europeo, al Consejo, al Comité Económico y Social Europeo y al Comité de las Regiones: El Pacto Verde Europeo, Bruselas, 11.12.2019 $\operatorname{COM}(2019) 640$ final.

${ }^{24}$ Dictamen del Comité Económico y Social Europeo sobre «La economía sostenible que necesitamos» (2020/C 106/01).

${ }^{25}$ Dictamen del Comité Económico y Social Europeo «Un planeta limpio para todos» (DO C 282 de 20.8.2019) y Dictamen del Comité Económico y Social Europeo «Justicia climática» (2018/C 081/04).
} 
respecto, prevé dar "prioridad a la dimensión humana y prestar atención a las regiones, los sectores y los trabajadores expuestos a los mayores desafios" 26.

El objetivo de la política industrial verde consiste en desencadenar y facilitar los cambios estructurales que conllevan o requieren, tanto para responder a las condiciones o situaciones ambientales, como para desarrollar una economía verde y $\operatorname{circular}^{27}$, definida la primera como una economía que mejora el bienestar humano y la equidad social, al tiempo que reduce significativamente los riesgos ambientales y la escasez ecológica $^{28}$, y la circular como aquella no lineal basada en el trinomio extraer-usar-ytirar, sino en la que desaparece el concepto de residuos y aparece el concepto de recursos, que pueden ser utilizados de nuevo por el sistema de producción ${ }^{29}$.

\subsection{Sectores e industrias implicadas en el Pacto Verde}

Si bien el plan de acción de la economía circular guiará la transición de todos los sectores, las actuaciones se centrarán en aquellos que hacen un uso intensivo de recursos, tales como el sector textil o los de la construcción ${ }^{30}$, la electrónica y los plásticos. Más detalladamente, el Pacto dibuja las siguientes prioridades, que afectarán de forma transversal a las siguientes actividades productivas ${ }^{31}$ :

1. Industria. En la hoja de ruta inicial de las políticas y medidas clave necesarias para hacer realidad el Pacto Verde Europeo y entre las medidas planeadas destaca la primera Ley del Clima europea. La meta señalada pasa por lograr la reducción de las emisiones de gases de efecto invernadero de aquí a 2030 al 50\%, como mínimo, y hacia el $55 \%$ con respecto a los niveles de 1990 de manera responsable. Asimismo, uno de los objetivos esenciales del nuevo marco político será estimular el desarrollo de mercados pioneros de productos climáticamente neutros y circulares, tanto dentro

\footnotetext{
${ }^{26}$ Comunicación de la Comisión al Parlamento Europeo, al Consejo Europeo, al Consejo, al Comité Económico y Social Europeo y al Comité de las Regiones: El Pacto Verde Europeo, Bruselas, 11.12.2019 $\operatorname{COM}(2019) 640$ final.

27 Sobre las fases y metodología para llevarla a cabo, United Nations Industrial Development Organization: Practitioner's Guide to Strategic Green Industrial Policy, 2016, p. 18.

28 United Nations Industrial Development Organization, Practitioner's Guide to Strategic Green Industrial Policy, cit., p. 18.

${ }^{29}$ Autores Varios, Informe sobre sostenibilidad en España 2017: cambio de rumbo, tiempo de acción, Fundación Alternativas, 2017, p. 37.

${ }^{30}$ Sobre la construcción en clave sostenible, RODRÍGUEZ ESCANCIANO, Susana, "Sostenibilidad ambiental y prevención de riesgos laborales: reflexiones sobre el sector de la construcción ecológica", Revista del Ministerio de Trabajo, Migraciones y Seguridad Social, núm. 138, 2018, p. 219 y ss.

${ }^{31}$ Un exhaustivo estudio sobre mitigación por sectores (energía, industria, transporte y construcción), Autores VArios, Roadmap to 2050. A Manual for Nations to Decarbonize by Mid-Century, Sustainable Development Solutions Network (SDSN) y Fundación Eni Enrico Mattei (FEEM), 2019.
} 
como fuera de la UE. Para dar respuesta a estos retos interrelacionados, la Comisión adoptará en 2021 un plan de acción "contaminación cero" para el aire, el agua y el suelo $^{32}$.

También el Parlamento Europeo pide "una ambiciosa Ley del Clima, con el objetivo jurídicamente vinculante y para todos los sectores de la economía de conseguir un nivel de cero emisiones netas de gases de efecto invernadero en 2050 a más tardar, así como con unos objetivos intermedios de la Unión para 2030 y 2040"33.

Esta nueva o renovada industria ha de ser sostenible, cualidad a aplicar tanto al proceso industrial como al producto, potenciando el uso eficiente de los recursos, la utilización optimizada de las materias primas y el adecuado tratamiento de los residuos; y también socialmente sostenible, apostando por trabajos dignos y por una protección social eficaz durante las transiciones.

2. Energía. Debe darse prioridad a la eficiencia energética mediante el desarrollo de un sector eléctrico basado en gran medida en fuentes renovables, completado con un rápido proceso de eliminación del carbón y con la descarbonización de gas ${ }^{34}$.

3. Tecnología. La Unión Europea (y el resto del mundo) se enfrenta al doble desafío de la transformación verde y digital y si bien la interacción de ambas puede conllevar aspectos positivos: "Europa debe aprovechar el potencial de la transformación digital en cuanto que factor clave para propiciar la consecución de los objetivos del Pacto Verde"35, no cabe olvidar la huella de carbono exponencialmente creciente de la industria tecnológica, similar al de la aviación ${ }^{36}$.

Desde el Derecho Laboral, cabe exigir que si la revolución tecnológica ha alumbrado una "industria inteligente", la misma ha de tener corazón "verde", esto

32 Comunicación de la Comisión al Parlamento Europeo, al Consejo Europeo, al Consejo, al Comité Económico y Social Europeo y al Comité de las Regiones: El Pacto Verde Europeo, Bruselas, 11.12.2019 $\operatorname{COM}(2019) 640$ final.

33 Resolución del Parlamento Europeo, de 15 de enero de 2020, sobre el Pacto Verde Europeo (2019/2956(RSP)).

${ }^{34}$ Comunicación de la Comisión al Parlamento Europeo, al Consejo Europeo, al Consejo, al Comité Económico y Social Europeo y al Comité de las Regiones: El Pacto Verde Europeo, Bruselas, 11.12.2019 $\operatorname{COM}(2019) 640$ final.

35 Comunicación de la Comisión al Parlamento Europeo, al Consejo Europeo, al Consejo, al Comité Económico y Social Europeo y al Comité de las Regiones: El Pacto Verde Europeo, Bruselas, 11.12.2019 $\operatorname{COM}(2019) 640$ final.

36 Crawford, Kate et al, AI Now 2019 Report. New York, AI Now Institute, 2019, https://ainowinstitute.org/AI_Now_2019_Report.html, p. 47. 
es, garantice la sostenibilidad y cree empleos sostenibles y decentes ${ }^{37}$ y viceversa, la ecologización de la economía y la transición acelerada a una economía baja en carbono requerirá el despliegue de una serie de tecnologías digitales ${ }^{38}$ : edificios inteligentes, planificación urbana inteligente y transporte inteligente, el teletrabajo y reuniones virtuales, etc. ${ }^{39}$.

En este aspecto, para la Comisión, "la tecnología supone un aliado en su lucha contra la contaminación y por ello explorará medidas que garanticen que las tecnologías digitales, tales como la inteligencia artificial, las redes 5G, la computación en la nube y en el borde y la internet de las cosas, puedan acelerar y potenciar los efectos de las políticas para combatir el cambio climático y proteger el medio ambiente. La digitalización brinda además nuevas oportunidades para el control a distancia de la contaminación del aire y del agua, o para la monitorización y optimización del modo de utilización de la energía y los recursos naturales". Para paliar las repercusiones negativas de su uso, prevé reforzar la transparencia sobre el impacto ambiental de los servicios de comunicaciones electrónicas, así como adoptar medidas más estrictas en el despliegue de redes nuevas y las ventajas de apoyar sistemas de recogida que incentiven la devolución de los dispositivos que no se quieran conservar, como teléfonos móviles, tabletas y cargadores $^{40}$.

4. Envases y plásticos. La Comisión hará un seguimiento de la estrategia de los plásticos de 2018 centrando la atención, entre otras cosas, en la adopción de medidas contra los microplásticos añadidos intencionadamente y contra la liberación no intencionada de plásticos en ámbitos como la producción textil o la abrasión de neumáticos ${ }^{41}$. Asimismo, elaborará una serie de requisitos para garantizar que todos los envases del mercado de la UE puedan reciclarse o reutilizarse de manera económicamente viable de aquí a 2030, establecerá un marco regulador para los

\footnotetext{
${ }^{37}$ Álvarez CuESTA, Henar, Empleos verdes: una aproximación desde el Derecho del Trabajo, cit. p. 21 y ss.

${ }^{38}$ Un análisis en Álvarez CUeSTA, Henar, "El empleo verde y la industria 4.0: hacia una transición justa" en $4^{a}$ Revolución Industrial: impacto de la automatización y la inteligencia artificial en la sociedad y la economía digital, Cizur Menor, Aranzadi, 2018, p. 633 y ss.

${ }^{39}$ COMITÉ ECONÓMICO Y SOCIAL, "Hacia un trabajo digital justo", 2016.

${ }^{40}$ Comunicación de la Comisión al Parlamento Europeo, al Consejo Europeo, al Consejo, al Comité Económico y Social Europeo y al Comité de las Regiones: El Pacto Verde Europeo, Bruselas, 11.12.2019 $\operatorname{COM}(2019) 640$ final.

${ }^{41}$ Sobre antiguos y nuevos contaminantes, Parliamentary Office of Science and Technology, Research for Parliament: Preparing for a changing world, UK Parliament, 2019, p. 56 y ss.
} 
plásticos biodegradables y los bioplásticos, y adoptará medidas sobre los plásticos de un solo uso ${ }^{42}$.

5. Reciclaje y reutilización. El plan de acción de la economía circular pretende incluir medidas para animar a las empresas a que ofrezcan productos reutilizables, duraderos y reparables y para que los consumidores puedan elegirlos; al tiempo, prevé analizar la necesidad de establecer un "derecho a la reparación" capaz de coto a la obsolescencia programada de los dispositivos, sobre todo en el sector de la electrónica ${ }^{43}$.

En este sentido, el Parlamento europeo apoya una legislación destinada a que los productos sean más duraderos, reparables, reutilizables y reciclables; pide que se presenten propuestas legislativas sobre el derecho a la reparación, la eliminación de la obsolescencia programada; y centra su atención en las baterías y dispositivos de carga comunes para equipos informáticos móviles ${ }^{44}$.

6. Transporte sostenible. El transporte representa la cuarta parte de las emisiones de gases de efecto invernadero de la Unión, y va en aumento. Para lograr la neutralidad climática $^{45}$, es necesaria una reducción del 90\% de las emisiones procedentes del transporte (por carretera, por ferrocarril, aéreo y por vías navegables) de aquí a 2050, y en su consecución, la Comisión se propone adoptar una estrategia de movilidad sostenible e inteligente en 2020 que acometerá este reto y abordará todas las fuentes de emisiones ${ }^{46}$.

\footnotetext{
${ }^{42}$ Comunicación de la Comisión al Parlamento Europeo, al Consejo Europeo, al Consejo, al Comité Económico y Social Europeo y al Comité de las Regiones: El Pacto Verde Europeo, Bruselas, 11.12.2019 $\operatorname{COM}(2019) 640$ final.

${ }^{43}$ Comunicación de la Comisión al Parlamento Europeo, al Consejo Europeo, al Consejo, al Comité Económico y Social Europeo y al Comité de las Regiones: El Pacto Verde Europeo, Bruselas, 11.12.2019 $\operatorname{COM}(2019) 640$ final.

${ }_{44}$ Resolución del Parlamento Europeo, de 15 de enero de 2020, sobre el Pacto Verde Europeo (2019/2956(RSP)).

${ }^{45}$ La Estrategia Europea a Largo Plazo "Un planeta limpio para todos", Comunicación de la Comisión al Parlamento Europeo, al Consejo Europeo, al Consejo, al Comité Económico y Social Europeo, al Comité de las Regiones y al Banco Europeo de Inversiones "Un planeta limpio para todos: La visión estratégica europea a largo plazo de una economía próspera, moderna, competitiva y climáticamente neutra" 28.11.2018 (COM/2018/773) propone una visión para conseguir de aquí a 2050 la neutralidad climática por medio de una transición socialmente justa y rentable.

${ }^{46}$ Comunicación de la Comisión al Parlamento Europeo, al Consejo Europeo, al Consejo, al Comité Económico y Social Europeo y al Comité de las Regiones: El Pacto Verde Europeo, Bruselas, 11.12.2019 $\operatorname{COM}(2019) 640$ final.
} 
7. Agricultura, ganadería y pesca. El Parlamento Europeo "destaca la importancia de la agricultura europea y su potencial para contribuir a la acción por el clima, la economía circular y a la mejora de la biodiversidad y para promover el uso sostenible de materias primas renovables; [y] destaca que los agricultores de la Unión deben recibir las herramientas necesarias para luchar contra el cambio climático y adaptarse al mismo"47. También el Consejo Económico y Social Europeo considera este sector como clave de bóveda ("la agricultura apoyada por la comunidad, las cadenas cortas de suministro, las redes alternativas de alimentación, los sistemas agrícolas locales, las ventas directas y las cooperativas de consumidores tienen el potencial de suministrar bienes públicos y proporcionar seguridad alimentaria, protección social y producción sostenible"48), pero ambas instituciones apuestan por otro modelo lejano, parece, de la explotación intensiva industrial, forzando a un nuevo reajuste en el mundo rural.

8. Industria química. Para garantizar un entorno sin sustancias tóxicas, la Comisión presentará una estrategia en el ámbito de las sustancias químicas con vistas a la sostenibilidad. Esta estrategia contribuirá tanto a mejorar la protección de los ciudadanos y el medio ambiente contra las sustancias químicas peligrosas como a impulsar la innovación para el desarrollo de alternativas seguras y sostenibles ${ }^{49}$.

\subsection{Dimensión social del Pacto Verde: la transición justa}

Entre las múltiples implicaciones que conllevan los cambios propuestos destaca la repercusión sobre los sectores mencionados en términos de empleo (por cuanto aquí importa, su calidad y cantidad).

Cierto es que las estimaciones son halagüeñas en términos netos: se destruirán puestos, pero se compensará con la creación de otros nuevos (de nuevo surge la comparación

\footnotetext{
47 "Destaca que la agricultura tiene el potencial para ayudar a la Unión a reducir sus emisiones mediante prácticas sostenibles, como la agricultura de precisión, la agricultura ecológica, la agroecología, la agrosilvicultura, el aumento del bienestar de los animales y la prevención de enfermedades humanas y animales, incluida la gestión sostenible de los bosques, la captura de carbono, y una mejor gestión de los nutrientes para contribuir a alcanzar los objetivos del Pacto Verde Europeo; recalca la importancia de incentivar a los agricultores para que adopten métodos que aporten beneficios cada vez mayores para el clima, el medio ambiente y la biodiversidad de manera justa, oportuna y económicamente viable", Resolución del Parlamento Europeo, de 15 de enero de 2020, sobre el Pacto Verde Europeo (2019/2956(RSP)).

${ }^{48}$ Dictamen del Comité Económico y Social Europeo sobre «La economía sostenible que necesitamos» (2020/C 106/01).

${ }^{49}$ Comunicación de la Comisión al Parlamento Europeo, al Consejo Europeo, al Consejo, al Comité Económico y Social Europeo y al Comité de las Regiones: El Pacto Verde Europeo, Bruselas, 11.12.2019 $\operatorname{COM}(2019) 640$ final.
} 
con el impacto de la robotización ${ }^{50}$ ). En términos generales, parece que el desarrollo y el amplio uso de tecnología con bajas emisiones de carbono creará empleos con salarios por encima de la media ${ }^{51}$. Por su parte, la OIT ha estimado que, en el marco de la transición hacia la sostenibilidad energética, "hasta 2030, se crearán casi 25 millones de empleos y se perderán 7 millones de empleos en todo el mundo"52 y "el desarrollo de una economía circular permitirían crear para 2030 un total neto entre 7 y 8 millones de nuevos empleos, con respecto a una situación en la que todo siguiera igual. En la hipótesis de que se verifique el escenario de la economía circular, se creará un volumen bruto de unos 78 millones de empleos, al tiempo que se destruirán alrededor de 71 millones. En lo que se refiere a los trabajadores que perderán el empleo, un número importante, cerca de 49 millones, encontrarán vacantes en la misma ocupación en otros sectores dentro del mismo país, es decir, a través de la reubicación de la fuerza de trabajo. En cuanto a los demás, se crearán cerca de 29 millones de empleos, sin reubicación, y se destruirán algo menos de 22 millones de empleos sin que se abran vacantes en la misma ocupación en otros sectores"53.

Pero las cifras no pueden ocultar que un número alto de personas trabajadoras perderán su empleo y tendrán serias dificultades para conseguir otra ocupación. Dicha situación se verá exacerbada dependiendo de la edad, formación, género y territorio en el que residan.

En consecuencia, y retornando a la idea vertebral, no cabe olvidar el impacto social de las medidas medioambientales, garantizando, como pide el Parlamento europeo, la "cohesión económica, social y territorial de las transiciones, prestando especial atención a las regiones más desfavorecidas, a las zonas afectadas por la transición industrial (principalmente las regiones mineras y las zonas dependientes de industrias con altas emisiones de carbono, como la producción de acero), a las zonas escasamente pobladas y a los territorios vulnerables desde el punto de vista medioambiental" 54 .

Para ello, y como parte del Plan de Inversiones para una Europa Sostenible, la Comisión propondrá "un Mecanismo para una Transición Justa, con un Fondo de Transición

\footnotetext{
${ }^{50} \mathrm{Al}$ respecto, RODRÍGUEZ FERNÁNDEZ, María Luz, Humanos y robots: empleo y condiciones de trabajo en la era tecnológica, Albacete, Bomarzo, 2020 o POQUET CATALÁ, Raquel, "Interrogantes abiertos de la robótica en el derecho del trabajo", Información Laboral, núm. 11, 2017, p. 71 y ss.

${ }^{51}$ Parlamento Europeo, The impact of climate change on the employment situation, 2010.

${ }^{52}$ OIT, Competencias profesionales para un futuro más ecológico: Conclusiones principales, Ginebra, OIT, 2019, p. 9.

${ }^{53}$ OIT, Competencias profesionales para un futuro más ecológico: Conclusiones principales, cit., p. 10.

${ }^{54}$ Resolución del Parlamento Europeo, de 15 de enero de 2020, sobre el Pacto Verde Europeo (2019/2956(RSP)).
} 
Justa, para que nadie se quede rezagado", el cual se centrará en aquellas regiones y sectores más afectados porque dependan de combustibles fósiles o de procesos intensivos en carbono. También tendrá por objetivo proteger a los ciudadanos y trabajadores más vulnerables a la transición, facilitándoles el acceso a programas de reciclaje profesional, empleo en nuevos sectores de la economía o viviendas eficientes desde el punto de vista energético ${ }^{55}$.

Como sucede con la revolución tecnológica, las medidas que facilitan un cambio socialmente aceptable y beneficioso en este caso pasan por el desarrollo de competencias adaptadas a las nuevas necesidades productivas (según estima la OIT "hará falta una inversión masiva en formación para que los trabajadores adquieran las competencias profesionales que se exigirán a quienes ocupen alrededor de 20 millones de nuevos empleos" ${ }^{\text {"56 }}$ ) y la protección social de quienes han perdido su puesto y no han podido acceder a los nuevos creados ${ }^{57}$.

Respecto a las primeras, en una economía de bajo carbono y con alta inversión en digitalización, es probable que algunas habilidades se vuelvan obsoletas debido a cambios estructurales en el mercado de trabajo y, al tiempo, se creen nuevas habilidades a medida que surjan nuevas ocupaciones de "cuello verde", y algunos puestos de trabajo existentes cambiarán de naturaleza ${ }^{58}$. Por ello, se necesitarán competencias más específicas relacionadas con la economía verde ${ }^{59}$, como el conocimiento de los materiales sostenibles, las capacidades para medir la "impresión de huella de carbono" y las habilidades de evaluación del impacto ambiental (flora y fauna) ${ }^{60}$. La estrategia ha de consistir en anticiparse a las tendencias futuras del empleo en estas economías verdes, con el fin de que los Estados puedan evitar tanto la desigualdad y la pobreza como la escasez de mano de obra ${ }^{61}$.

En todo caso, en la formulación de las mismas ha de tenerse en cuenta la variable de género existente ("los empleos verdes pueden suponer tanto una oportunidad como un

55 Comunicación de la Comisión al Parlamento Europeo, al Consejo Europeo, al Consejo, al Comité Económico y Social Europeo y al Comité de las Regiones: El Pacto Verde Europeo, Bruselas, 11.12.2019 $\operatorname{COM}(2019) 640$ final.

${ }^{56}$ OIT, Competencias profesionales para un futuro más ecológico: Conclusiones principales, cit., p. 9.

${ }^{57}$ OIT, Work in a changing climate, The Green Initiative, Ginebra, OIT, 2017, p. 13.

58 Sobre el futuro del trabajo (y el diseño de un nuevo Estatuto de los Trabajadores) y la necesaria reformulación de la formación "tecnológica", MERCADER UGUINA, Jesús, "Nuevos escenarios para el Estatuto de los Trabajadores del siglo XXI: digitalización y cambio tecnológico", Trabajo y Derecho, núm. 63, 2020, pp. 12 y ss.

${ }^{59} \mathrm{Al}$ respecto, OIT, Competencias profesionales para un futuro más ecológico, cit., p. 9 y ss.

${ }^{60}$ Parlamento Europeo, The impact of climate change on the employment situation, 2010.

${ }^{61}$ StePhenson, Sean, “Jobs, Justice, Climate: Conflicting State Obligations in the International Human Rights and Climate Change Regimes”, Ottawa Law Review, Vol. 42, núm. 1, 2010, p. 11. 
obstáculo en el objetivo de alcanzar la igualdad en el empleo entre mujeres y hombres" ${ }^{62}$ ), mientras los hombres con ocupaciones de calificación media tendrán una mayor necesidad de reciclarse profesionalmente, las mujeres obtendrán sólo una fracción de los puestos de trabajo creados ${ }^{63}$.

En cuanto a la protección social, han surgido propuestas destinadas a compensar a los trabajadores que pierdan su empleo o tengan dificultades en obtenerlo, bien por la lucha contra el cambio climático, bien por la digitalización de la actividad que han desarrollado. Para ellos los Estados deben crear políticas de reactivación diseñadas atendiendo a las características propias de cada sector y territorio ${ }^{64}$ y mientras tanto proporcionar adecuada protección social, no faltando voces que apuntan en la dirección de reforzar la protección por desempleo ${ }^{65}$, regular una renta básica universal a estos efectos $^{66}$, o medidas similares al recientemente aprobado ingreso mínimo vital regulado en el RD-Ley 20/2020, de 29 de mayo.

En fin, no faltan propuestas que, con la finalidad de hacer frente a la crisis climática y a la probable disminución de puestos de trabajo, opten por limitar la jornada de trabajo y disminuir la máxima prevista para facilitar el reparto del empleo ${ }^{67}$.

Para conseguir un adecuado diseño y la anticipación necesaria en la transición justa pretendida, la herramienta oportuna (como en tantas ocasiones) pasa por un "activo diálogo social", en tanto "contribuye a que empresas y trabajadores anticipen el cambio y lo gestionen con éxito" 68 , que ponga el foco en los "trabajadores de todos los sectores y comunidades de la Unión más afectados por la descarbonización, [y en] las regiones mineras con elevada intensidad en emisiones de carbono, a realizar la transición hacia una economía limpia del futuro, sin que ello tenga un efecto desalentador con respecto

\footnotetext{
62 Álvarez Cuesta, Henar, "Descubrir nuevos yacimientos de empleo: emprendimiento, empleos verdes, deporte, cultura y ocio", en La empleabilidad y la calidad en el empleo: apostando por la igualdad efectiva, Valencia, Tirant lo Blanch, 2019, p. 42 y ss.

${ }^{63}$ OIT, Competencias profesionales para un futuro más ecológico: Conclusiones principales, cit., p. 9.

${ }^{64}$ StePHEnson, Sean, "Jobs, Justice, Climate: Conflicting State Obligations in the International Human Rights and Climate Change Regimes", cit., p. 11.

${ }^{65}$ OIT, Sostenibilidad medioambiental con empleo, Perspectivas sociales y del empleo en el mundo 2018, Ginebra, OIT, 2018, pp. 41 y ss.

${ }^{66}$ Por todos, CARrizosa Prieto, Esther, "Hacia la articulación de una renta básica ciudadana en el ordenamiento jurídico español”, Revista Española de Derecho del Trabajo, núm. 192, 2016.

${ }^{67}$ FREY, Philipp, The Ecological Limits of Work: on carbon emissions, carbon budgets and working time, Hampshire, Autonomy, 2019.

${ }^{68}$ Comunicación de la Comisión al Parlamento Europeo, al Consejo Europeo, al Consejo, al Comité Económico y Social Europeo y al Comité de las Regiones: El Pacto Verde Europeo, Bruselas, 11.12.2019 $\operatorname{COM}(2019) 640$ final.
} 
a iniciativas y proyectos proactivos" ${ }^{69}$. A tal fin, el Fondo Social Europeo+ propuesto desempeñará un importante papel para ayudar a los trabajadores europeos a adquirir las competencias que necesitan para pasar de sectores en declive a sectores en expansión y adaptarse a nuevos procesos y la Agenda de Capacidades y la Garantía Juvenil se actualizarán para mejorar la empleabilidad en la economía verde ${ }^{70}$.

También en un segundo nivel de actuación, la negociación colectiva ha de incorporar como contenido propio (al menos en aquellos sectores y empresas más afectados por las medidas contenidas en el Pacto analizado y en las futuras normas de aplicación) mecanismos para diseñar los cambios productivos derivados de la transición justa pretendida, similares a los previstos respecto a la digitalización y automatización ("cada vez más los interlocutores sociales han de volcar su atención en la regulación de los despidos [climáticos], teniendo en consideración las características de la actividad o de la empresa en la que se aplique el convenio, pactando tiempo de adaptación, recolocación o superiores indemnizaciones" ${ }^{\prime 71}$ ), y prestando especial atención a las necesidades formativas creadas por el cambio a una economía verde.

\section{La lucha contra el cambio climático en España}

La hoja de ruta marcada desde la Unión Europea ha de ser seguida por los distintos países que forman parte de la misma, aun cuando varios Estados europeos ya han tomado medidas al respecto desde hace décadas, como Francia ${ }^{72}$, Gran Bretaña o Alemania $^{73}$.

En España, pese a contar con varios instrumentos destinados a tal fin, todavía no cuenta con una norma jurídica que sirva de marco, a la espera de la promulgación del Proyecto de Ley de Cambio Climático y Transición Energética. En él se recogen las líneas de tendencia diseñadas por el Pacto Verde Europeo, tanto en cuanto hace a las propuestas climáticas como respecto a la asunción del concepto de transición justa ya desde su

69 Resolución del Parlamento Europeo, de 15 de enero de 2020, sobre el Pacto Verde Europeo (2019/2956(RSP)).

${ }^{70}$ Comunicación de la Comisión al Parlamento Europeo, al Consejo Europeo, al Consejo, al Comité Económico y Social Europeo y al Comité de las Regiones: El Pacto Verde Europeo, Bruselas, 11.12.2019 $\operatorname{COM}(2019) 640$ final.

71 Álvarez Cuesta, Henar, "El diálogo social y la negociación colectiva como herramientas para lograr una transición digital justa”, Lan Harremanak, núm. 42, 2019, p. 22.

72 Rapport de Conseil d'Orientation pour l'Emploi, Croissance verte et emploi. France, January 2010, http://www.ladocumentationfrancaise.fr/rapports-publics/104000053/index.shtml.

73 Autores Varios, A growth strategy for Germany, New jobs through investments in energy and environment, 2009, $\quad$ http://www.bundesfinanzministerium.de/Content/DE/Downloads/G20Dokumente/Hamburg_Wachstumsstrategien/GER-Growth-Strategy.pdf?_blob=publicationFile\&v=3. 
Preámbulo: "España debe ofrecer respuestas solidarias e inclusivas a los colectivos más afectados por el cambio climático y la transformación de la economía... [; se] presenta una oportunidad desde el punto de vista económico y de modernización de nuestro país, así como desde el punto de vista social, facilitando la distribución equitativa de la riqueza en el proceso de descarbonización".

La norma proyectada se articula conforme al "principio de no regresión en los objetivos marcados", definido como aquel en virtud del cual "la normativa, la actividad de las administraciones públicas y la práctica jurisdiccional no pueden implicar una rebaja o un retroceso cuantitativo ni cualitativo respecto de los niveles de protección ambiental existentes en cada momento, salvo situaciones plenamente justificadas basadas en razones de interés público, y una vez realizado un juicio de ponderación entre los diferentes bienes jurídicos que pudieran entrar en contradicción con el ambiental" y crea, de conformidad con el Reglamento 2018/1999 del Parlamento Europeo y del Consejo, de 11 de diciembre de 2018, sobre la gobernanza de la Unión de la Energía y de la Acción por el Clima, los Planes Nacionales Integrados de Energía y Clima (PNIEC) y la Estrategia de Descarbonización a 2050, así como contempla el Plan Nacional de Adaptación al Cambio Climático (PNACC) como instrumento de coordinación.

El objeto, de conformidad con su artículo 1, se articula en cuatro pilares: asegurar el cumplimiento de los objetivos del Acuerdo de París, firmado por España el 22 de abril de 2016; facilitar la descarbonización de la economía española; promover la adaptación a los impactos del cambio climático; y, por último, implantar un modelo de desarrollo sostenible que genere empleo decente. Y como principios rectores recogidos en el artículo 2, conviene destacar, por cuanto aquí importa, los siguientes:

a. Desarrollo sostenible.

b. Descarbonización de la economía española, entendiendo por tal la consecución de un modelo socioeconómico sin emisiones de gases de efecto invernadero.

c. Protección del medio ambiente, y aplicación del principio "quien contamina, paga".

d. Cohesión social y territorial.

e. Resiliencia.

f. Protección y promoción de la salud pública.

g. Protección de colectivos vulnerables, con especial consideración a la infancia. h) Igualdad entre mujeres y hombres.

El mencionado Plan Nacional de Adaptación al Cambio Climático (PNACC) constituye el instrumento de planificación básico para promover la acción coordinada y coherente frente a los efectos del cambio climático en España. En él se definen los objetivos, 
criterios, ámbitos de aplicación y acciones para fomentar la resiliencia y la adaptación frente al cambio climático incluyendo (artículo 15):

a. La elaboración de escenarios climáticos regionalizados para la geografía española.

b. La recopilación, análisis y difusión de información acerca de la vulnerabilidad y adaptación al cambio climático en diferentes sectores socioeconómicos, sistemas ecológicos y territorios.

c. La promoción y coordinación de la participación de todos los agentes implicados en las políticas de adaptación, incluyendo los distintos niveles de la administración, las organizaciones sociales y la ciudadanía en su conjunto.

d. La definición de un sistema de indicadores de impactos y adaptación al cambio climático, que facilite un seguimiento y evaluación de las políticas públicas al respecto.

e. La elaboración de informes periódicos de seguimiento y evaluación del PNACC y sus programas de trabajo.

También el artículo 4 prevé el diseño de un Plan Nacional Integrado de Energía y Clima como herramienta de planificación estratégica nacional que integra la política de energía y clima e influirá en el futuro de las industrias afectadas.

Como objetivos concretos a alcanzar relacionados con las emisiones contaminantes y la energía empleada, para el año 2030 se pretende (artículo 3):

a. Reducir en el año 2030 las emisiones de gases de efecto invernadero del conjunto de la economía española en, al menos, un 20\% respecto del año 1990.

b. Alcanzar en el año 2030 una penetración de energías de origen renovable en el consumo de energía final de, al menos, un 35\%.

c. Alcanzar en el año 2030 un sistema eléctrico con, al menos, un 70\% de generación a partir de energías de origen renovable.

d. Mejorar la eficiencia energética disminuyendo el consumo de energía primaria en, al menos, un $35 \%$, con respecto a la línea de base conforme a normativa comunitaria.

La necesidad de alcanzar los porcentajes marcados a nivel europeo e interno forzará a todos los sectores de actividad a adaptar sus modos de producción (aun cuando resulta preciso diferenciar las consecuencias y estrategias a seguir en cada uno). Y una de las variables más afectadas va a ser el empleo, como ya se advirtió a nivel supranacional, si bien es cierto que se puede configurar, como hace el Marco Estratégico de Energía y Clima, como una oportunidad para la modernización de la economía española y la creación de empleo. En este sentido, considera que las medidas que se prevé adoptar generarán entre 250.000 y 364.000 nuevas ocupaciones de calidad entre 2021 y 2030 
(empleo anual no acumulado) respecto a un escenario en el que no se ponen en marcha estas medidas.

Por su parte, el Impacto económico, de empleo, social y sobre la salud pública del Borrador del Plan Nacional Integrado de Energía y Clima 2021-2030 de febrero de 2019 señala que el empleo neto aumentaría entre $250.000-364.000$ empleos/año $(+1.7 \%$ en 2030) y la tasa de paro se reduciría entre un $1,1 \%$ y un $1,6 \%$. Estos nuevos trabajos provendrían de las nuevas inversiones en renovables, ahorro y eficiencia y redes y, a partir de 2025, del efecto derivado del cambio energético. De este modo, "las inversiones en renovables generarian entre 102.000 y 182.000 empleos/año, mientras que las inversiones en ahorro y eficiencia energética generarian entre 42.000 y 80.000 empleos/año. Las inversiones en redes generarían entre 23.000 y 44.000 empleos/año. El cambio energético generaría indirectamente hasta 173.000 empleos/año en 2030"74.

Sin embargo, las cifras mostradas no pueden enmascarar los puestos en riesgo debido a la transformación que han de acometer todas las empresas (en mayor o menor medida). En consecuencia, esta "transición a una economía descarbonizada requiere también de medidas que faciliten una transición justa para los colectivos y áreas geográficas más vulnerables" (Proyecto de Ley del Cambio Climático). En aras a lograr la sostenibilidad social pretendida, el artículo 24 del Proyecto regula la Estrategia de Transición Justa como "el instrumento de ámbito estatal dirigido a la optimización de las oportunidades en la actividad y el empleo de la transición hacia una economía baja en emisiones de gases de efecto invernadero y a la identificación y adopción de medidas que garanticen un tratamiento equitativo y solidario a trabajadores y territorios en dicha transición", aprobadas por el Gobierno, cada cinco años, con la participación de las Comunidades Autónomas y los agentes sociales, e incluirá los siguientes contenidos, teniendo en cuenta la perspectiva de género:

a. Identificación de colectivos, sectores y territorios potencialmente vulnerables al proceso de transición a una economía baja en emisiones de carbono.

b. Análisis de las oportunidades de creación de actividad económica y empleo vinculadas a la transición energética.

c. Políticas industriales, de investigación y desarrollo, de innovación, de promoción de actividad económica y de empleo y formación ocupacional para la transición justa.

d. Instrumentos para el seguimiento del mercado de trabajo en el marco de la transición energética mediante la participación de los agentes sociales, como en las mesas de diálogo social.

e. El marco de elaboración de los convenios de Transición Justa.

\footnotetext{
${ }^{74}$ Borrador del Plan Nacional Integrado de Energía y Clima 2021-2030 de febrero de 2019.
} 
En el contexto de dicha Estrategia se suscribirán convenios de transición justa con el objeto fomentar la actividad económica y su modernización, así como la búsqueda o mejora de la empleabilidad de trabajadores vulnerables a la transición hacia una economía baja en emisiones de carbono, en particular, en casos de cierre o reconversión de instalaciones. Se suscribirán entre el Ministerio para la Transición Ecológica y el Reto Demográfico, previo informe del Ministerio de Trabajo y Economía Social, del Ministerio Inclusión, Seguridad Social y Migraciones y del Ministerio de Industria, Turismo y Comercio, y otras Administraciones públicas, en particular, entidades locales de áreas geográficas vulnerables a la transición hacia una economía baja en carbono y podrán participar empresas, organizaciones de los sectores empresariales, organizaciones sindicales, universidades, centros educativos, asociaciones y organizaciones ambientales no gubernamentales y demás entidades interesadas o afectadas (artículo 25).

El contenido de estos convenios incluirá (artículo 25.3):

a. Una evaluación del estado de vulnerabilidad del área geográfica o colectivo afectado.

b. Compromisos de las partes participantes en el convenio, incluidas las empresas beneficiarias de medidas de apoyo para la transición.

c. Medidas fiscales, de financiación, de apoyo a la $\mathrm{I}+\mathrm{D}+\mathrm{i}$, de empleo, de protección social y actividades formativas para incentivar la adaptación de los trabajadores, supeditadas al cumplimiento de los objetivos establecidos en el convenio.

d. Un calendario para la adopción de las medidas, con objetivos medibles y mecanismos de seguimiento.

e. También podrán incluir el acceso prioritario a una parte o a la totalidad de la capacidad de evacuación eléctrica, así como el derecho prioritario al uso y volumen de agua de aquellas concesiones que queden extinguidas tras el cierre de instalaciones de generación de energía eléctrica con arreglo a lo previsto en el Real Decreto-ley 17/2019, de 22 de noviembre, por el que se adoptan medidas urgentes para la necesaria adaptación de parámetros retributivos que afectan al sistema eléctrico y por el que se da respuesta al proceso de cese de actividad de centrales térmicas de generación.

En cuanto a su vigencia, vendrá determinada en las cláusulas del propio convenio, no pudiendo superar en ningún caso los siete años de duración inicial, prorrogables por los firmantes, antes de la finalización del plazo de vigencia previsto, por un período adicional 7 años (artículo 25.4). 
Precursor de esta herramienta (y también de los ajustes derivados de la apuesta por una energía más limpia), el Acuerdo Marco para una Transición Justa de la Minería del Carbón y Desarrollo Sostenible de las Comarcas Mineras para el período 2019-2027 firmado el 24 de octubre de 2018 (y las ayudas sociales que recoge el RD-Ley 25/2018, de 21 de diciembre, de medidas urgentes para una transición justa de la minería del carbón y el desarrollo sostenible de las comarcas mineras) marca la senda de los convenios antes mencionados.

Recoge como medida específica la inversión en formación de los trabajadores en los nuevos sectores y prevé especial atención a los empleados de las subcontratas ("en los planes de restauración, energía renovable, eficiencia energética y contratos de transición se empleará también preferentemente a dichos trabajadores" ${ }^{75}$. El objetivo final de estas medidas es la "reactivación económica y desarrollo alternativo de las comarcas mineras para lograr su transformación estructural, recuperación económica y bienestar social... Igualmente se persigue atenuar el impacto que produce la pérdida de puestos de trabajo y las repercusiones del cierre de aquellas unidades de producción de carbón que no puedan o no deseen continuar en la economía regional. Dada la elevada concentración de las minas de carbón en España se trata, en definitiva, de abordar los efectos perjudiciales que el cierre de las minas de carbón que no continúen a partir del 2019 puede provocar en los mercados laborales regionales, con una posible sobrecarga de parados del sector minero".

Los errores en la planificación de los cambios desarrollados en las cuencas mineras en las últimas décadas han de llevar a la reflexión sobre las estrategias a utilizar para conseguir la transición justa pretendida en aquellas empresas pertenecientes a los sectores enumerados en las páginas precedentes y a cuantas organizaciones productivas dependan de los mismos, que tendrán que comenzar a anticipar los cambios.

Entre las herramientas a utilizar, conviene prestar especial atención al diálogo social con sindicatos y empresas implicadas en las transformaciones pretendidas, comenzar el diseño con la debida anticipación con el fin de lograr formar en las nuevas competencias a cuantas personas prestan servicios en los sectores afectados (directa o indirectamente y sin olvidar a las empresas contratistas y subcontratistas) y desarrollar nuevos modelos de producción o sectores de actividad en los territorios afectados, muchos de ellos dependientes de una sola industria o empresa.

Por último, la pandemia causada por la COVID19 repercutirá en la lucha contra el cambio climático, pues el terremoto que ha supuesto para la sociedad en su conjunto

75 Sobre transición justa en las cuencas mineras, GALGÓcZI, Béla, "Phasing out coal: a just transition approach", Working Paper European Trade Union Institute, núm. 4, 2019, pp. 30 y ss. 
puede variar los objetivos asumidos y la financiación pretendida. Además, puede también transformar los modos y maneras de trabajar, viajar y consumir en un sentido positivo (con menores desplazamientos y un consumo local y responsable) y en negativo (mayor necesidad energética por mor de la tecnología empleada, aún mayor dependencia del plástico, menor utilización del transporte colectivo y abuso de bienes de un solo uso). Todo ello habrá de tenerse en consideración a la hora de especificar y desarrollar tanto el Pacto Verde europeo como el Proyecto de Ley español.

\section{Conclusiones}

Frente al cambio climático, hablar de desafíos o retos a veces puede acabar por banalizar sus consecuencias, tanto las que acarrea la inacción contra el mismo como las derivadas de los mecanismos de lucha, y entre ellas cabe incluir las laborales.

Respuestas como las analizadas proporcionadas por la Unión Europea o la bosquejada por España señalan el camino y hacen necesario (una necesidad cada vez más acuciante), diseñar herramientas para alcanzar la transición justa pretendida. Cerrar los ojos a las implicaciones laborales que conllevan no van a hacer que desaparezcan, pero estudiar el impacto que presentan en cada sector o industria (señalados y conocidos los objetivos de reducción y eliminación a alcanzar) va a permitir anticipar la medida adecuada en cada caso.

En primer lugar, y pese a las distintas cifras de puestos "perdidos" y "creados", no se puede ocultar que un número alto de personas trabajadoras perderán su empleo y tendrán serias dificultades para conseguir otra ocupación y dicha situación se verá exacerbada dependiendo de la edad, formación, género y territorio en el que residan.

En segundo término, la transición justa pretendida lleva aparejada una apuesta por el empleo verde o los ecoempleos intrínsecamente unidos a unas condiciones laborales justas y dignas. La transición a una economía verde no puede obviar la necesaria vinculación con el trabajo decente.

En tercero, es preciso luchar también, y de nuevo, contra la brecha de género que pueden llevar aparejadas estas ocupaciones altamente técnicas en las industrias verdes, en tanto pueden reproducir la existente en el ámbito tecnológico.

Para llevar a cabo la transición justa pretendida (uniendo empleo verde y trabajo decente en la lucha contra el cambio climático) la labor de los agentes sociales resulta imprescindible. De un lado, el diálogo social, tanto a nivel bipartito como tripartito, es una de las principales herramientas para lograr dotar de una adecuada perspectiva social 
a las leyes contra el cambio climático y diseñar la protección social necesaria para quienes más sufran las consecuencias del mismo, pese a los fallos en la aplicación de los acuerdos alcanzados; a los defectos en la formación y escasa financiación; o a la falta de coordinación y cooperación entre los diferentes agentes colectivos implicados.

De otro, a través de la negociación colectiva en todos los niveles las organizaciones sindicales y empresariales han de convertirse (lo están haciendo ya) en agentes de defensa del medio ambiente y de lucha contra el cambio climático. En los sectores y empresas más afectados por los objetivos previstos en el Pacto Verde europeo (y por la futura Ley española) se ha de comenzar a negociar los mecanismos para paliar las consecuencias y aprovechar las ventajas. Los acuerdos alcanzados han de contribuir a evitar la pérdida de empleos, propiciar el reciclaje de las personas trabajadoras a la vista de la estrategia empresarial adaptada a las nuevas coordenadas socioeconómicas y asegurar unas condiciones laborales dignas (incluyendo la prevención de los riesgos laborales y la igualdad).

En fin, no cabe olvidar cómo la pandemia causada por la COVID19 repercutirá en la lucha contra el cambio climático, pues el terremoto que ha supuesto para la sociedad en su conjunto puede variar los objetivos asumidos y la financiación pretendida, pero no cabe dar un paso atrás frente al cambio climático, al contrario, ha de servir para profundizar en la construcción sostenible, social e integradora tanto de la Unión Europea como de España.

\section{Bibliografía}

Álvarez Cuesta, Henar, Empleos verdes. Una aproximación desde el Derecho del Trabajo, Albacete, Bomarzo, 2016.

Álvarez Cuesta, Henar, "Descubrir nuevos yacimientos de empleo: emprendimiento, empleos verdes, deporte, cultura y ocio", en La empleabilidad y la calidad en el empleo: apostando por la igualdad efectiva, Valencia, Tirant lo Blanch, 2019.

Álvarez Cuesta, Henar, "El contenido social en las leyes contra el cambio climático", en La Gouvernance et l'enjeu de concilier l'èthique et la performance, Pamplona, Eunsa, 2018.

Álvarez Cuesta, Henar, "El empleo verde y la industria 4.0: hacia una transición justa" en $4^{a}$ Revolución Industrial: impacto de la automatización y la inteligencia artificial en la sociedad y la economía digital, Cizur Menor, Aranzadi, 2018. 
Autores Varios, A growth strategy for Germany, New jobs through investments in energy and environment, 2009, http://www.bundesfinanzministerium.de/Content/DE/Downloads/G20-

Dokumente/Hamburg_Wachstumsstrategien/GER-Growth-

Strategy.pdf?_blob=publicationFile \&v $=3$.

Autores Varios, Cambio climático 2014 Impactos, adaptación y vulnerabilidad. Contribución del Grupo de trabajo II al Quinto Informe de Evaluación del Grupo Intergubernamental de Expertos sobre el Cambio Climático, 2014.

Autores Varios, Climate change and labour: impacts of heat in the workplace, UNDP, 2016.

Autores Varios, Informe sobre sostenibilidad en España 2017: cambio de rumbo, tiempo de acción, Fundación Alternativas, 2017.

Autores Varios, Roadmap to 2050. A Manual for Nations to Decarbonize by MidCentury, Sustainable Development Solutions Network (SDSN) y Fundación Eni Enrico Mattei (FEEM), 2019.

CARRIZOSA PRIETO, Esther, "Hacia la articulación de una renta básica ciudadana en el ordenamiento jurídico español”, Revista Española de Derecho del Trabajo, núm. 192, 2016.

ChacARtegui Jávega, Consuelo, Negociación colectiva y sostenibilidad medioambiental: un compromiso social y ecológico, Albacete, Bomarzo, 2018.

Comisión Europea, Comunicación de la Comisión al Parlamento Europeo, al Consejo Europeo, al Consejo, al Comité Económico y Social Europeo, al Comité de las Regiones y al Banco Europeo de Inversiones "Un planeta limpio para todos: La visión estratégica europea a largo plazo de una economía próspera, moderna, competitiva y climáticamente neutra” 28.11.2018 (COM/2018/773) final.

Comisión Europea, Comunicación de la Comisión al Parlamento Europeo, al Consejo Europeo, al Consejo, al Comité Económico y Social Europeo y al Comité de las Regiones: El Pacto Verde Europeo, Bruselas, 11.12.2019 COM(2019) 640 final.

Comité Económico y Social, "Hacia un trabajo digital justo", 2016. 
Comité Económico y Social, Dictamen del Comité Económico y Social Europeo «Justicia climática» (2018/C 081/04).

Comité Económico y Social, Dictamen del Comité Económico y Social Europeo «Un planeta limpio para todos» (DO C 282 de 20.8.2019).

Comité Económico y Social, Dictamen del Comité Económico y Social Europeo sobre «La economía sostenible que necesitamos» (2020/C 106/01).

Crawford, Kate et alii, AI Now 2019 Report. New York, AI Now Institute, 2019, https://ainowinstitute.org/AI Now 2019 Report.html.

Doorey, David, "Just Transitions Law: Putting Labour Law to Work on Climate Change", Journal of Environmental Law and Practice, núm. 30, 2017.

DUPRESSOIR, Sophie et alii, Climate Change and employment: Impact on employment in the European Union-25 of climate change and CO2 emission reduction measures by 2030, Brussels, Wuppertal Institute, 2007.

FREY, Phillip, The Ecological Limits of Work: on carbon emissions, carbon budgets and working time, Hampshire, Autonomy, 2019.

GALGÓCZI, Béla, "Phasing out coal: a just transition approach", Working Paper European Trade Union Institute, núm. 4, 2019.

Instituto Sindical de Trabajo, Ambiente y Salud (ISTAS), ¿Cómo afecta el cambio climático a la salud humana?, CC.OO./ISTAS, 2016.

LÓPEZ RodríGuez, Josune, "The promotion of both decent and Green jobs through cooperatives", Boletín de la Asociación Internacional de Derecho Cooperativo, núm. $54,2019$.

McKinsey Global Institute, Climate risk and response. Physical hazards and socioeconomic impacts, enero 2020.

MerCAder Uguina, Jesús, "Nuevos escenarios para el Estatuto de los Trabajadores del siglo XXI: digitalización y cambio tecnológico”, Trabajo y Derecho, núm. 63, 2020.

Monereo Pérez, José Luis, y Rivas VAlLejo, Pilar, Prevención de riesgos laborales y medio ambiente, Granada, Comares, 2008. 
OIT, Competencias profesionales para un futuro más ecológico: Conclusiones principales, Ginebra, OIT, 2019.

OIT, Directrices de politica para una transición justa hacia economías y sociedades ambientalmente sostenibles para todos, Ginebra, OIT, 2015.

OIT, Guidelines for a just transition towards environmentally sustainable economies and societies for all, Ginebra, OIT, 2015.

OIT, Sostenibilidad medioambiental con empleo, Perspectivas sociales y del empleo en el mundo 2018, Ginebra, OIT, 2018.

OIT, The social and decent work dimensions of a new Agreement on Climate Change, Ginebra, OIT, 2009.

OIT, Time to act for SDG 8. Integrating Decent Work, Sustained Growth and Environmental Integrity, Ginebra, OIT, 2019.

OIT, Work in a changing climate, The Green Initiative, Ginebra, OIT, 2017.

Parlamento Europeo, The impact of climate change on the employment situation, 2010.

Parlamento Europeo, Resolución del Parlamento Europeo, de 15 de enero de 2020, sobre el Pacto Verde Europeo (2019/2956(RSP)).

Parliamentary Office of Science and Technology, Research for Parliament: Preparing for a changing world, UK Parliament, 2019.

PÉREZ AMORÓs, Francisco, "Derecho del trabajo y medio ambiente: unas notas introductorias", Gaceta Laboral, vol. 14, 2010.

Plan Nacional de Adaptación al Cambio Climático 2021-2030 Borrador 30 abril 2020.

Poquet CATAlÁ, Raquel, "Interrogantes abiertos de la robótica en el derecho del trabajo”, Información Laboral, núm. 11, 2017.

QuiNTERo LimA, María Gemma, “ODS 8: trabajo decente y el futuro del trabajo, Tiempo de paz, núm. 132, 2019. 
Rapport de Conseil d'Orientation pour l'Emploi, Croissance verte et emploi. France, January 2010, $\quad$ http://www.ladocumentationfrancaise.fr/rapportspublics/104000053/index.shtml.

RODRÍGUEZ ESCANCIANO, Susana, "Sostenibilidad ambiental y prevención de riesgos laborales: reflexiones sobre el sector de la construcción ecológica", Revista del Ministerio de Trabajo, Migraciones y Seguridad Social, núm. 138, 2018.

Rodríguez FERnÁndez, María Luz, Humanos y robots: empleo y condiciones de trabajo en la era tecnológica, Albacete, Bomarzo, 2020.

RODRÍGUEZ, María Luz, "El diálogo social y la negociación colectiva como herramientas para lograr una transición digital justa", Lan Harremanak, núm. 42, 2019.

Rosemberg, Anabella, "Llevar a cabo una transición justa. Las conexiones entre el cambio climático y el empleo, y propuestas para futuras investigaciones", Boletín Internacional de Investigación Sindical, núm. 2, vol. 2, 2010.

StePhenson, Sean, "Jobs, Justice, Climate: Conflicting State Obligations in the International Human Rights and Climate Change Regimes", Ottawa Law Review, Vol. 42, núm. 1, 2010.

United Nations Industrial Development Organization, Practitioner's Guide to Strategic Green Industrial Policy, 2016. 